\title{
Hilbert space factorization and Fourier type of operators
}

by

\author{
Aicke Hinrichs (Jena and College Station, TX)
}

\begin{abstract}
It is an open question whether every Fourier type 2 operator factors through a Hilbert space. We show that at least the natural gradations of Fourier type 2 norms and Hilbert space factorization norms are not uniformly equivalent. A corresponding result is also obtained for a number of other sequences of ideal norms instead of the Fourier type 2 gradation including the Walsh function analogue of Fourier type. Our main tools are ideal norms and random matrices.
\end{abstract}

1. Introduction. Let $\mathcal{F}$ denote the Fourier transform on the real line given by

$$
\mathcal{F} f(s)=\frac{1}{\sqrt{2 \pi}} \int_{-\infty}^{\infty} f(t) e^{-i s t} d t
$$

$X$ and $Y$ are always complex Banach spaces. $\mathcal{L}(X, Y)$ denotes the Banach space of bounded linear operators from $X$ to $Y$. An operator $T \in \mathcal{L}(X, Y)$ is said to be of Fourier type $p$ for some $p \in(1,2]$ if the operator $\mathcal{F} \otimes T$, which is, by the classical Hausdorff-Young inequality, a well defined operator between the algebraic tensor products $L_{p}(\mathbb{R}) \otimes X$ and $L_{q}(\mathbb{R}) \otimes Y$, has a bounded linear extension acting between the vector-valued spaces $L_{p}^{X}(\mathbb{R})$ and $L_{q}^{Y}(\mathbb{R})$. Here $q$ is the conjugate number of $p$ given by $1 / p+1 / q=1$. We say that $X$ has Fourier type $p$ if the identity operator $I_{X}$ does.

The notion of Fourier type was introduced by Peetre [Pee] in the case of Banach spaces. He studied interpolation properties of such spaces. Kwapień [Kwa] proved that $X$ has Fourier type 2 if and only if $X$ is isomorphic to a Hilbert space. The deepest result in this direction is due to Bourgain [Bou1], [Bou2] who showed that $X$ has some Fourier type $p>1$ if and only if it is B-convex, i.e. has some Rademacher type $p>1$.

Bourgain's theorem fails for operators, i.e. there exist operators of Rademacher type 2 failing to have any Fourier type $p>1$ (see $[\mathrm{PW}]$ ). The

2000 Mathematics Subject Classification: 47L20, 47B10, 47D50.

Key words and phrases: Fourier type, factorization, random matrices.

Supported by DFG grant PI 584/2-1. 
operator case of Kwapien's result is still an open question. It is not known whether every operator of Fourier type 2 factors through a Hilbert space (see $[\mathrm{DF}],[\mathrm{PW}])$. It is the main purpose of this paper to shed some light onto this problem.

Both notions of Fourier type and factorization through a Hilbert space are local notions in the sense that they depend only on the finite-dimensional pieces of the operator under consideration. This is made precise in the following two theorems. Proofs of both of them can be found in $[\mathrm{PW}]$. Both theorems are mainly due to Kwapień [Kwa] (see also [Koe] and [Pis]).

THEOREM 1.1. For any $T \in \mathcal{L}(X, Y)$ the following assertions are equivalent.

(i) $T$ has Fourier type 2.

(ii) There exists $c>0$ such that for all sequences $x_{1}, x_{2}, \ldots$ in $X$,

$$
\int_{0}^{1}\left\|\sum_{k=1}^{\infty} \exp (2 \pi i k t) T x_{k}\right\|^{2} d t \leq c^{2} \sum_{k=1}^{\infty}\left\|x_{k}\right\|^{2} .
$$

(iii) There exists $c>0$ such that for all $n \in \mathbb{N}$ and $x_{1}, \ldots, x_{n} \in X$,

$$
\sum_{h=1}^{n}\left\|\sum_{k=1}^{n} \frac{1}{\sqrt{n}} \exp (2 \pi i h k / n) T x_{k}\right\|^{2} \leq c^{2} \sum_{k=1}^{n}\left\|x_{k}\right\|^{2} .
$$

THEOREM 1.2. For any $T \in \mathcal{L}(X, Y)$ the following assertions are equivalent.

(i) $T$ factors through a Hilbert space, i.e. there exists a Hilbert space $H$ and operators $B \in \mathcal{L}(X, H)$ and $A \in \mathcal{L}(H, Y)$ such that $T=A B$.

(ii) There exists $c>0$ such that for all orthonormal systems $\left(a_{1}, a_{2}, \ldots\right)$ in some Hilbert space $L_{2}(M, \mu)$ and all sequences $x_{1}, x_{2}, \ldots$ in $X$,

$$
\int_{M}\left\|\sum_{k=1}^{\infty} a_{k}(t) T x_{k}\right\|^{2} d \mu(t) \leq c^{2} \sum_{k=1}^{\infty}\left\|x_{k}\right\|^{2} .
$$

(iii) There exists $c>0$ such that for all $n \in \mathbb{N}, n \times n$-matrices $A_{n}=\left(\alpha_{h k}\right)$ satisfying $\left\|A_{n}: l_{2}^{n} \rightarrow l_{2}^{n}\right\| \leq 1$ and $x_{1}, \ldots, x_{n} \in X$,

$$
\sum_{h=1}^{n}\left\|\sum_{k=1}^{n} \alpha_{h k} T x_{k}\right\|^{2} \leq c^{2} \sum_{k=1}^{n}\left\|x_{k}\right\|^{2} \text {. }
$$

REMARK. A characterization similar to Theorem 1 is possible for Fourier type $p$ operators (see $[\mathrm{PW}])$. We restricted ourselves to the case $p=2$ to make the similarity between both theorems more striking.

For a fixed $n \times n$-matrix $A_{n}$ and $T \in \mathcal{L}(X, Y)$, define $\kappa\left(T \mid A_{n}\right)$ as the smallest constant $c$ such that $(2)$ holds for all $x_{1}, \ldots, x_{n} \in X$. The functional 
$\kappa\left(A_{n}\right): T \rightarrow \kappa\left(T \mid A_{n}\right)$ is the Kwapien ideal norm associated with $A_{n}$. The universal Kwapien ideal norm of $T$ is defined as

$$
\kappa_{n}(T)=\sup \left\{\kappa\left(T \mid A_{n}\right):\left\|A_{n}: l_{2}^{n} \rightarrow l_{2}^{n}\right\| \leq 1\right\} .
$$

For the general theory of ideal norms associated with matrices and orthonormal systems and, in particular, for properties of the ideal norms just defined we refer the reader to $[\mathrm{PW}]$. Observe that Theorem 1.2 says that the sequence $\left(\kappa_{n}\right)$ of ideal norms is a natural gradation in the sense of [Tom] of the Hilbert space factorization norm generally defined as inf $\|A\| \cdot\|B\|$ where the infimum is taken over all factorizations $T=A B$ as in (i). Similarly, if we let $E_{n}=(\exp (2 \pi i h k / n) / \sqrt{n})$, then Theorem 1.1 states that $\left(\kappa\left(E_{n}\right)\right)$ is a natural gradation of the Fourier type 2 norm, which may be defined as the operator norm of $\mathcal{F} \otimes T: L_{2}^{X}(\mathbb{R}) \rightarrow L_{2}^{Y}(\mathbb{R})$.

Considering the problem whether every Fourier type 2 operator factors through a Hilbert space it is natural to ask whether the sequences of ideal norms $\kappa_{n}$ and $\kappa\left(E_{n}\right)$ are uniformly equivalent, i.e. whether there exists a constant $c$ such that $\kappa_{n}(T) \leq c \kappa\left(T \mid E_{n}\right)$ for all operators $T$. The following theorem shows that this is not true.

THEOREM 1.3. For any sequence $\left(A_{n}\right)$ of $n \times n$-matrices which satisfies $\left\|A_{n}: l_{2}^{n} \rightarrow l_{2}^{n}\right\| \leq 1$ there exists a sequence $\left(T_{n}\right)$ of operators such that

$$
\kappa_{n}\left(T_{n}\right)=1 \quad \text { and } \quad \lim _{n \rightarrow \infty} n^{\alpha} \kappa\left(T_{n} \mid A_{n}\right)=0 \quad \text { for } \alpha<1 / 10 .
$$

This theorem also answers Problem 2.3.16 in [PW] about the existence of universal matrices for Kwapien ideal norms. We even show that sets of matrices needed to approximate $\kappa_{n}(T)$ for all $T$ up to some fixed constant factor (independent of $n$ ) must have big size. See Theorem 5.2 for a detailed statement.

The proof of Theorem 1.3 is nonconstructive. We use operators $T_{n}$ : $l_{1}^{n} \rightarrow l_{\infty}^{n}$ whose representing matrices have random entries of modulus 1 . In Section 2, we clarify the conditions under which such an operator can have large value of $\kappa\left(T_{n} \mid A_{n}\right)$ for a fixed $n \times n$-matrix $A_{n}$. Section 3 contains an estimate for the probability that this happens, which will be shown to be negligible. In Section 4, we derive from the results of [BGN] about the norm of such random matrices that the probability of having $\kappa_{n}\left(T_{n}\right)$ large is almost 1. This is used to prove Theorems 1.3 and 5.2. Finally, applications to the behavior of ideal norms associated with the trigonometric system and the Walsh system are contained in Section 6.

2. Operators from $l_{1}^{n}$ to $l_{\infty}^{n}$. The purpose of this section is to provide necessary conditions for an operator in $\mathcal{L}\left(l_{1}^{n}, l_{\infty}^{n}\right)$ to have large $\kappa\left(T \mid A_{n}\right)$ for a fixed matrix $A_{n}$. By a $c$-net $\mathcal{N}$ in a metric space $M$ we mean a subset of $M$ such that the balls of radius $c$ with centers in $\mathcal{N}$ cover $M$. 
Proposition 2.1. Let $T \in \mathcal{L}\left(l_{1}^{n}, l_{\infty}^{n}\right)$ be an operator induced by a matrix $\left(\tau_{i j}\right)$. Let $A_{n}=\left(\alpha_{h k}\right)$ be an $n \times n$-matrix with $\left\|A_{n}: l_{2}^{n} \rightarrow l_{2}^{n}\right\| \leq 1$. For some $c>0$, let $\mathcal{N}$ be a c/8-net in $B_{l_{2}^{n}} \cap \frac{2}{c \sqrt{n}} B_{l_{\infty}^{n}}$ in $l_{2}^{n}$-distance. If $\kappa\left(T \mid A_{n}\right) \geq c \sqrt{n}\|T\|$, then there exists a partition $\{1, \ldots, n\}=\bigcup_{i \in F} M_{i}$ and $\lambda=\left(\lambda_{1}, \ldots, \lambda_{n}\right) \in \mathcal{N}$ such that

(i) $F \subset\{1, \ldots, n\}, 1 \leq\left|M_{i}\right| \leq 16 / c^{2}$ for $i \in F$ and

(ii) $\sum_{h=1}^{n} \max _{1 \leq j \leq n}\left|\sum_{i \in F}\left(\sum_{k \in M_{i}} \lambda_{k} \alpha_{h k}\right) \tau_{j i}\right|^{2} \geq \frac{c^{2} n\|T\|^{2}}{64}$.

Proof. Because of homogeneity we can assume that $T$ is a norm one operator, that is, $\left|\tau_{i j}\right| \leq 1$ for $i, j=1, \ldots, n$. Let $e_{1}, \ldots, e_{n}$ denote the unit vectors in $l_{1}^{n}$. An extreme point argument (see [PW], p. 22) shows that

$$
\kappa\left(T \mid A_{n}\right)^{2}=\sum_{h=1}^{n}\left\|\sum_{k=1}^{n} \mu_{k} T e_{\varphi(k)} \alpha_{h k}\right\|^{2}
$$

for some map $\varphi:\{1, \ldots, n\} \rightarrow\{1, \ldots, n\}$ and numbers $\mu_{1}, \ldots, \mu_{n}$ with $\sum_{k=1}^{n}\left|\mu_{k}\right|^{2}=1$. Let

$$
A=\left\{k \in\{1, \ldots, n\}:\left|\mu_{k}\right| \leq 2 /(c \sqrt{n})\right\} \quad \text { and } \quad A^{\mathrm{c}}=\{1, \ldots, n\} \backslash A .
$$

Then $1=\sum_{k=1}^{n}\left|\mu_{k}\right|^{2} \geq\left(4 /\left(c^{2} n\right)\right)\left|A^{\mathrm{c}}\right|$ implies $\left|A^{\mathrm{c}}\right| \leq c^{2} n / 4$. It follows from (3) and the assumption $\kappa\left(T \mid A_{n}\right) \geq c \sqrt{n}$ that

$$
\begin{aligned}
c^{2} n & \leq \sum_{h=1}^{n}\left\|\sum_{k=1}^{n} \mu_{k} T e_{\varphi(k)} \alpha_{h k}\right\|^{2} \\
& \leq 2 \sum_{h=1}^{n}\left\|\sum_{k \in A} \mu_{k} T e_{\varphi(k)} \alpha_{h k}\right\|^{2}+2 \sum_{h=1}^{n}\left\|\sum_{k \in A^{\mathrm{c}}} \mu_{k} T e_{\varphi(k)} \alpha_{h k}\right\|^{2} .
\end{aligned}
$$

Now the bound on the cardinality of $A^{\mathrm{c}},\|T\| \leq 1$, and $\left\|A_{n}: l_{2}^{n} \rightarrow l_{2}^{n}\right\| \leq 1$ imply that

$$
\begin{aligned}
& \sum_{h=1}^{n}\left\|\sum_{k \in A^{\mathrm{c}}} \mu_{k} T e_{\varphi(k)} \alpha_{h k}\right\|^{2} \leq \sum_{h=1}^{n}\left(\sum_{k \in A^{\mathrm{c}}}\left|\mu_{k}\right| \cdot\left|\alpha_{h k}\right|\right)^{2} \\
& \leq \sum_{h=1}^{n}\left(\sum_{k \in A^{\mathrm{c}}}\left|\mu_{k}\right|^{2}\right)\left(\sum_{k \in A^{\mathrm{c}}}\left|\alpha_{h k}\right|^{2}\right) \leq \sum_{k \in A^{\mathrm{c}}} \sum_{h=1}^{n}\left|\alpha_{h k}\right|^{2} \leq\left|A^{\mathrm{c}}\right| \leq c^{2} n / 4 .
\end{aligned}
$$

Hence

$$
c^{2} n / 4 \leq \sum_{h=1}^{n}\left\|\sum_{k \in A} \mu_{k} T e_{\varphi(k)} \alpha_{h k}\right\|^{2}
$$


We define the level sets $M_{i}=\{k \in A: \varphi(k)=i\}$ for $i=1, \ldots, n$. Then

$$
c^{2} n / 4 \leq \sum_{h=1}^{n}\left\|\sum_{i=1}^{n}\left(\sum_{k \in M_{i}} \mu_{k} \alpha_{h k}\right) T e_{i}\right\|^{2}=\sum_{h=1}^{n} \max _{1 \leq j \leq n}\left|\sum_{i=1}^{n} \sum_{k \in M_{i}} \mu_{k} \alpha_{h k} \tau_{j i}\right|^{2} \text {. }
$$

Let

$$
B=\left\{i \in\{1, \ldots, n\}:\left|M_{i}\right| \leq 16 / c^{2}\right\} \quad \text { and } \quad B^{\mathrm{c}}=\{1, \ldots, n\} \backslash B .
$$

We conclude from the preceding inequality that

$$
\begin{aligned}
c^{2} n / 4 \leq & 2 \sum_{h=1}^{n} \max _{1 \leq j \leq n}\left|\sum_{i \in B} \sum_{k \in M_{i}} \mu_{k} \alpha_{h k} \tau_{j i}\right|^{2} \\
& +2 \sum_{h=1}^{n} \max _{1 \leq j \leq n}\left|\sum_{i \in B^{\mathrm{c}}} \sum_{k \in M_{i}} \mu_{k} \alpha_{h k} \tau_{j i}\right|^{2} .
\end{aligned}
$$

Since the sets $M_{i}$ form a partition of $A$, we find that

$$
n \geq|A|=\sum_{i=1}^{n}\left|M_{i}\right| \geq \frac{16}{c^{2}}\left|B^{\mathrm{c}}\right|
$$

which in turn yields $\left|B^{\mathrm{c}}\right| \leq c^{2} n / 16$. Using again the assumptions $\left|\tau_{j i}\right| \leq 1$ and $\left\|A_{n}: l_{2}^{n} \rightarrow l_{2}^{n}\right\| \leq 1$, we derive that

$$
\begin{aligned}
\sum_{h=1}^{n} \max _{1 \leq j \leq n}\left|\sum_{i \in B^{\mathrm{c}}} \sum_{k \in M_{i}} \mu_{k} \alpha_{h k} \tau_{j i}\right|^{2} \leq \sum_{h=1}^{n}\left(\sum_{i \in B^{\mathrm{c}}}\left|\sum_{k \in M_{i}} \mu_{k} \alpha_{h k}\right|\right)^{2} \\
\leq\left|B^{\mathrm{c}}\right| \sum_{h=1}^{n} \sum_{i \in B^{\mathrm{c}}}\left|\sum_{k \in M_{i}} \mu_{k} \alpha_{h k}\right|^{2} \leq \frac{c^{2} n}{16} \sum_{i \in B^{\mathrm{c}}} \sum_{k \in M_{i}}\left|\mu_{k}\right|^{2} \leq \frac{c^{2} n}{16} .
\end{aligned}
$$

Hence we arrive at

$$
c^{2} n / 16 \leq \sum_{h=1}^{n} \max _{1 \leq j \leq n}\left|\sum_{i \in B} \sum_{k \in M_{i}} \mu_{k} \alpha_{h k} \tau_{j i}\right|^{2}
$$

which is nearly what we want. In the last step of the proof we replace $\left(\mu_{k}\right)$ by $\left(\lambda_{k}\right) \in \mathcal{N}$. To this end we define

$$
\lambda_{k}^{o}= \begin{cases}\mu_{k} & \text { if } k \in M_{i} \text { for some } i \in B \\ 0 & \text { otherwise. }\end{cases}
$$

We extend $B$ to a set $F$ and define $M_{i}$ for $i \in F \backslash B$ such that $\left(M_{i}\right)$ forms a partition of $\{1, \ldots, n\}$ with $\left|M_{i}\right| \leq 16 / c^{2}$ for all $i \in F$. Removing the empty $M_{i}$ 's we may and do assume that $|F| \leq n$ and $F \subset\{1, \ldots, n\}$. Then we can rewrite inequality (4) as

$$
c^{2} n / 16 \leq \sum_{h=1}^{n} \max _{1 \leq j \leq n}\left|\sum_{i \in F} \sum_{k \in M_{i}} \lambda_{k}^{o} \alpha_{h k} \tau_{j i}\right|^{2}
$$


Our construction yields $\left(\lambda_{1}^{o}, \ldots, \lambda_{n}^{o}\right) \in B_{l_{2}^{n}} \cap \frac{2}{c \sqrt{n}} B_{l_{\infty}^{n}}$. Thus we can find $\lambda=\left(\lambda_{1}, \ldots, \lambda_{n}\right) \in \mathcal{N}$ such that

$$
\sum_{k=1}^{n}\left|\lambda_{k}^{o}-\lambda_{k}\right|^{2} \leq c^{2} / 64 .
$$

Now it follows from (5) that

$$
\begin{aligned}
c^{2} n / 16 \leq & 2 \sum_{h=1}^{n} \max _{1 \leq j \leq n}\left|\sum_{i \in F} \sum_{k \in M_{i}} \lambda_{k} \alpha_{h k} \tau_{j i}\right|^{2} \\
& +2 \sum_{h=1}^{n} \max _{1 \leq j \leq n}\left|\sum_{i \in F} \sum_{k \in M_{i}}\left(\lambda_{k}^{o}-\lambda_{k}\right) \alpha_{h k} \tau_{j i}\right|^{2} .
\end{aligned}
$$

Finally, we use once more the assumptions $\left|\tau_{j i}\right| \leq 1$ and $\left\|A_{n}: l_{2}^{n} \rightarrow l_{2}^{n}\right\| \leq 1$ to conclude that

$$
\begin{aligned}
& \sum_{h=1}^{n} \max _{1 \leq j \leq n}\left|\sum_{i \in F} \sum_{k \in M_{i}}\left(\lambda_{k}^{o}-\lambda_{k}\right) \alpha_{h k} \tau_{j i}\right|^{2} \leq \sum_{h=1}^{n}\left(\sum_{i \in F}\left|\sum_{k \in M_{i}}\left(\lambda_{k}^{o}-\lambda_{k}\right) \alpha_{h k}\right|\right)^{2} \\
& \leq|F| \sum_{i \in F} \sum_{h=1}^{n}\left|\sum_{k \in M_{i}}\left(\lambda_{k}^{o}-\lambda_{k}\right) \alpha_{h k}\right|^{2} \leq n \sum_{i \in F} \sum_{k \in M_{i}}\left|\lambda_{k}^{o}-\lambda_{k}\right|^{2} \leq c^{2} n / 64
\end{aligned}
$$

which in turn implies the claimed property (ii).

REMARK. The proposition is sharp in the following sense. If there exist a partition $\{1, \ldots, n\}=\bigcup_{i \in F} M_{i}$ and $\lambda=\left(\lambda_{1}, \ldots, \lambda_{n}\right) \in B_{l_{2}^{n}}$ such that (ii) holds then $\kappa\left(T \mid A_{n}\right) \geq c \sqrt{n}\|T\| / 8$. This can be seen directly from the definition of $\kappa\left(T \mid A_{n}\right)$ by choosing $x_{k}=\lambda_{k} e_{i}$ for $k \in M_{i}$.

3. Random matrices. Let $\mathcal{M}_{n}$ be the set of all $n \times n$-matrices with entries \pm 1 , viewed as operators in $\mathcal{L}\left(l_{1}^{n}, l_{\infty}^{n}\right)$, equipped with the normalized counting measure $P$, that is, $P(T)=2^{-n^{2}}$ for all $T \in \mathcal{M}_{n}$. The next theorem which will be the main tool in the following sections shows that the probability of $\kappa\left(T: l_{1}^{n} \rightarrow l_{\infty}^{n} \mid A_{n}\right)$ being large is very small for a fixed $n \times n$-matrix $A_{n}$. In contrast, the probability of $T$ factoring nicely through a Hilbert space is also very small. As we will see, this follows quite easily from well known norm estimates for random matrices. Consequently, for any given $n \times n$-matrix $A_{n}$ with $\left\|A_{n}: l_{2}^{n} \rightarrow l_{2}^{n}\right\| \leq 1$, there must be $n \times n$-matrices $T$ with \pm 1 entries which have, as operators in $\mathcal{L}\left(l_{1}^{n}, l_{\infty}^{n}\right)$, large $\kappa_{n}(T)$ but considerably smaller $\kappa\left(T \mid A_{n}\right)$. We now exploit this approach in detail.

Theorem 3.1. For $n=1,2, \ldots$, every $n \times n$-matrix $A_{n}$ satisfying $\left\|A_{n}: l_{2}^{n} \rightarrow l_{2}^{n}\right\| \leq 1$ and $1 / \sqrt{n} \leq c \leq 1$, we have

$$
\mathbb{P}\left(\kappa\left(T \mid A_{n}\right) \geq c \sqrt{n}\right) \leq 2^{-2^{-33} c^{10} n^{2}+4 n\left(4+\log _{2} n\right)} .
$$


Proof. We fix a $c / 8$-net $\mathcal{N}$ in $B_{l_{2}^{n}} \cap \frac{2}{c \sqrt{n}} B_{l_{\infty}^{n}}$ in $l_{2}^{n}$-distance. Let $\mathcal{M}_{n}^{o}$ be the set of all $T \in \mathcal{M}_{n}$ satisfying the conclusion of Proposition 2.1. That means that for each $T \in \mathcal{M}_{n}^{o}$ there exist a partition $\left(M_{i}\right)_{i \in F}$ of $\{1, \ldots, n\}$ with nonempty $M_{i}$ and $\lambda \in \mathcal{N}$ with properties (i) and (ii). Proposition 2.1 tells us that $\mathbb{P}\left(\kappa\left(T \mid A_{n}\right) \geq c \sqrt{n}\right) \leq \mathbb{P}\left(\mathcal{M}_{n}^{o}\right)$. Let $\mathcal{P}$ be the collection of all partitions $\left(M_{i}\right)_{i \in F}$ of $\{1, \ldots, n\}$ satisfying (i). For fixed $P \in \mathcal{P}$ and $\lambda \in \mathcal{N}$, we define $\mathcal{M}(P, \lambda)=\left\{T \in \mathcal{M}_{n}\right.$ : (ii) is also satisfied $\}$. Then it follows from

$$
\mathcal{M}_{n}^{o}=\bigcup_{P \in \mathcal{P}} \bigcup_{\lambda \in \mathcal{N}} \mathcal{M}(P, \lambda)
$$

that

$$
\mathbb{P}\left(\mathcal{M}_{n}^{o}\right) \leq \sum_{P \in \mathcal{P}} \sum_{\lambda \in \mathcal{N}} \mathbb{P}(\mathcal{M}(P, \lambda)) .
$$

The key lemma to be proved below is as follows.

Lemma 3.2. For all $P \in \mathcal{P}$ and $\lambda \in \mathcal{N}$, we have

$$
\mathbb{P}(\mathcal{M}(P, \lambda)) \leq 2^{-2^{-33} c^{10} n^{2}+2 n\left(1+\log _{2} n\right) .}
$$

Together with the above considerations, this lemma now implies

$$
\mathbb{P}\left(\kappa\left(T \mid A_{n}\right) \geq c \sqrt{n}\right) \leq|\mathcal{P}| \cdot|\mathcal{N}| 2^{-2^{-33} c^{10} n^{2}+2 n\left(1+\log _{2} n\right)} .
$$

It remains to estimate the cardinalities of $\mathcal{P}$ and $\mathcal{N}$. A trivial bound on the cardinality of $\mathcal{P}$ is

$$
|\mathcal{P}| \leq n^{n} \leq 2^{n\left(1+\log _{2} n\right)} .
$$

Moreover, it follows from well known entropy estimates that $\mathcal{N}$ can be chosen such that

$$
|\mathcal{N}| \leq(17 / c)^{2 n} \leq 2^{2 n \log _{2}(17 / c)} .
$$

Since we assumed $c \geq 1 / \sqrt{n}$, this implies

$$
|\mathcal{N}| \leq 2^{2 n \log _{2}(17 \sqrt{n})} \leq 2^{n\left(10+\log _{2} n\right)} .
$$

Now (6)-(8) provide the claimed estimate in the theorem.

Proof of Lemma 3.2. For $h=1, \ldots, n$, we define

$$
N_{h}=\left\{\varepsilon \in\{+1,-1\}^{F}:\left|\sum_{i \in F}\left(\sum_{k \in M_{i}} \lambda_{k} \alpha_{h k}\right) \varepsilon_{i}\right| \geq c / 16\right\} .
$$

First, we observe that, for any $\varepsilon \in\{+1,-1\}^{F}$, the set $A(\varepsilon)=\{h \in$ $\left.\{1, \ldots, n\}: \varepsilon \in N_{h}\right\}$ satisfies the cardinality estimate

$$
|A(\varepsilon)| \leq 256 / c^{2} .
$$


Indeed, this follows from $\left\|A_{n}: l_{2}^{n} \rightarrow l_{2}^{n}\right\| \leq 1$ since

$$
|A(\varepsilon)| \frac{c^{2}}{256} \leq \sum_{h=1}^{n}\left|\sum_{i \in F}\left(\sum_{k \in M_{i}} \lambda_{k} \alpha_{h k}\right) \varepsilon_{i}\right|^{2} \leq \sum_{i \in F} \sum_{k \in M_{i}}\left|\lambda_{k}\right|^{2} \leq 1 .
$$

Let now $T \in \mathcal{M}(P, \lambda)$ and define

$$
B=\left\{h \in\{1, \ldots, n\}: \max _{1 \leq j \leq n}\left|\sum_{i \in F}\left(\sum_{k \in M_{i}} \lambda_{k} \alpha_{h k}\right) \tau_{j i}\right| \geq c / 16\right\}
$$

and $B^{\mathrm{c}}=\{1, \ldots, n\} \backslash B$. Then

$$
\sum_{h \in B^{\mathrm{c}}} \max _{1 \leq j \leq n}\left|\sum_{i \in F}\left(\sum_{k \in M_{i}} \lambda_{k} \alpha_{h k}\right) \tau_{j i}\right|^{2} \leq\left|B^{\mathrm{c}}\right| \frac{c^{2}}{256}=\frac{c^{2}}{256}(n-|B|)
$$

and

$$
\begin{aligned}
\sum_{h \in B} \max _{1 \leq j \leq n}\left|\sum_{i \in F}\left(\sum_{k \in M_{i}} \lambda_{k} \alpha_{h k}\right) \tau_{j i}\right|^{2} & \leq \sum_{h \in B}\left(\sum_{k=1}^{n}\left|\lambda_{k}\right| \cdot\left|\alpha_{h k}\right|\right)^{2} \\
& \leq \sum_{h \in B}\left(\sum_{k=1}^{n}\left|\lambda_{k}\right|^{2}\right)\left(\sum_{k=1}^{n}\left|\alpha_{h k}\right|^{2}\right) \leq|B| .
\end{aligned}
$$

Now it follows from the assumed property (ii) that

$$
\frac{c^{2} n}{64} \leq|B|+(n-|B|) \frac{c^{2}}{256} \leq \frac{c^{2} n}{256}+|B|,
$$

which implies $|B| \geq c^{2} n / 128$. Let

$$
B_{j}=\left\{h \in\{1, \ldots, n\}:\left|\sum_{i \in F}\left(\sum_{k \in M_{i}} \lambda_{k} \alpha_{h k}\right) \tau_{j i}\right| \geq c / 16\right\}=A\left(\left(\tau_{j i}\right)_{i}\right) .
$$

Then $B=\bigcup_{j=1}^{n} B_{j}$. Furthermore, let $N=\bigcup_{h=1}^{n} N_{h}$ and

$$
C=\left\{j \in\{1, \ldots, n\}:\left(\tau_{j i}\right)_{i=1}^{n} \in N\right\}=\left\{j \in\{1, \ldots, n\}: B_{j} \neq \emptyset\right\} .
$$

Then it follows from (9) that

$$
\frac{c^{2} n}{128} \leq|B| \leq \sum_{j=1}^{n}\left|B_{j}\right|=\sum_{j \in C}\left|B_{j}\right| \leq \frac{256}{c^{2}}|C|,
$$

which gives $|C| \geq c^{4} n / 2^{15}$.

Let $\mathcal{C}$ denote the collection of all subsets $C \subset\{1, \ldots, n\}$ satisfying this cardinality estimate. For $C \in \mathcal{C}$, let

$$
\mathcal{T}(C)=\left\{T \in \mathcal{M}(P, \lambda):\left(\tau_{j i}\right)_{i=1}^{n} \in N \text { for } j \in C\right\} .
$$

The above considerations tell us that $\bigcup_{C \in \mathcal{C}} \mathcal{T}(C)=\mathcal{M}(P, \lambda)$, which implies that

$$
\mathbb{P}(\mathcal{M}(P, \lambda)) \leq \sum_{C \in \mathcal{C}} \mathbb{P}(\mathcal{T}(C))
$$


To estimate $\mathbb{P}(\mathcal{T}(C))$, we have to estimate the size of $N$. It follows from the well known tail behavior estimate of Rademacher series with real coefficients that if $\varepsilon_{1}, \ldots, \varepsilon_{m}$ are independent random variables identically distributed with $\mathbb{P}\left(\varepsilon_{k}=+1\right)=\mathbb{P}\left(\varepsilon_{k}=-1\right)=1 / 2$ and $\zeta_{1}, \ldots, \zeta_{m} \in C$, then

$$
\mathbb{P}\left(\left|\sum \varepsilon_{k} \zeta_{k}\right|>\delta\right) \leq 4 \exp \left(-\frac{\delta^{2}}{4 \sum\left|\zeta_{k}\right|^{2}}\right) \quad \text { for } \delta>0
$$

Using this, we see from the definition of $N_{h}$ that

$$
\left|N_{h}\right| \leq 2^{|F|+2} \exp \left(-\frac{2^{-10} c^{2}}{\sum_{i \in F}\left|\sum_{k \in M_{i}} \lambda_{k} \alpha_{h k}\right|^{2}}\right) .
$$

Moreover, we conclude from $\left|M_{i}\right| \leq 16 / c^{2}$ for $i \in F$ and $\left|\lambda_{k}\right| \leq 2 / c \sqrt{n}$ that

$$
\text { (11) } \begin{aligned}
\sum_{i \in F}\left|\sum_{k \in M_{i}} \lambda_{k} \alpha_{h k}\right|^{2} & \leq \sum_{i \in F}\left(\sum_{k \in M_{i}}\left|\lambda_{k}\right| \cdot\left|\alpha_{h k}\right|\right)^{2} \leq \frac{4}{c^{2} n} \sum_{i \in F}\left(\sum_{k \in M_{i}}\left|\alpha_{h k}\right|\right)^{2} \\
& \leq \frac{4}{c^{2} n} \sum_{i \in F}\left|M_{i}\right| \sum_{k \in M_{i}}\left|\alpha_{h k}\right|^{2} \leq \frac{64}{c^{4} n} \sum_{k=1}^{n}\left|\alpha_{h k}\right|^{2} \leq \frac{64}{c^{4} n .}
\end{aligned}
$$

Together with $|F| \leq n$ this implies

$$
\left|N_{h}\right| \leq 2^{n+2} \exp \left(-2^{-18} c^{6} n\right)=2^{n\left(1-2^{-18} c^{6} \log _{2} e\right)+2}
$$

and

$$
|N| \leq \sum_{h=1}^{n}\left|N_{h}\right| \leq 2^{n\left(1-2^{-18} c^{6}\right)+2+\log _{2} n} .
$$

Now we are able to estimate the size of $\mathcal{T}(C)$ as

$$
\begin{aligned}
|\mathcal{T}(C)| & \left.\leq|N|^{|C|}\left(2^{n}\right)^{n-|C|} \leq 2^{n\left(1-2^{-18}\right.} c^{6}\right)|C|+\left(2+\log _{2} n\right)|C|+n^{2}-n|C| \\
& \leq 2^{n^{2}-2^{-18} c^{6} n|C|+n\left(2+\log _{2} n\right)} \leq 2^{n^{2}\left(1-2^{-33} c^{10}\right)+n\left(2+\log _{2} n\right)} .
\end{aligned}
$$

Hence

$$
\mathbb{P}(\mathcal{T}(C)) \leq 2^{-2^{-33} c^{10} n^{2}+n\left(2+\log _{2} n\right)} .
$$

Finally, observing that $|\mathcal{C}| \leq n^{n}=2^{n \log _{2} n}$, an appeal to (10) finishes the proof.

REMARK. If $A_{n}$ is a unimodular orthogonal $n \times n$-matrix, which means that $\left|\alpha_{h k}\right|=1 / \sqrt{n}$ for $h, k=1, \ldots, n$, the estimate (11) can be improved to

$$
\sum_{i \in F}\left|\sum_{k \in M_{i}} \lambda_{k} \alpha_{h k}\right|^{2} \leq \frac{1}{n} \sum_{i \in F}\left(\sum_{k \in M_{i}}\left|\lambda_{k}\right|\right)^{2} \leq \frac{1}{n} \sum_{i \in F}\left|M_{i}\right| \sum_{k \in M_{i}}\left|\lambda_{k}\right|^{2} \leq \frac{16}{c^{2} n} .
$$

This yields a better estimate in Theorem 3.1 for such matrices, in fact the fraction $2^{-33} c^{10}$ can be replaced by $2^{-31} c^{8}$.

The next corollary is an immediate consequence of Theorem 3.1. 
Corollary 3.3. Let $c>0$ and let $\left(A_{n}\right)_{n \geq 1}$ be a sequence of $n \times n$ matrices with $\left\|A_{n}: l_{2}^{n} \rightarrow l_{2}^{n}\right\| \leq 1$. Then $\mathbb{P}\left(\kappa\left(T \mid A_{n}\right) \geq c \sqrt{n}\right) \rightarrow 0$ as $n \rightarrow \infty$.

This corollary can be made more quantitative by keeping track of the constants.

Corollary 3.4. Let $\left(A_{n}\right)_{n \geq 1}$ be a sequence of $n \times n$-matrices with $\left\|A_{n}: l_{2}^{n} \rightarrow l_{2}^{n}\right\| \leq 1$. Then $\mathbb{P}\left(\kappa\left(T \mid A_{n}\right) \geq n^{\alpha}\right) \rightarrow 0$ as $n \rightarrow \infty$ for any $\alpha>2 / 5$.

Proof. Setting $c_{n}=n^{\alpha-1 / 2}$ and applying Theorem 3.1 gives

$$
\mathbb{P}\left(\kappa\left(T \mid A_{n}\right) \geq n^{\alpha}\right)=\mathbb{P}\left(\kappa\left(T \mid A_{n}\right) \geq c_{n} \sqrt{n}\right) \leq 2^{-2^{-33} n^{10 \alpha-3}+4 n\left(4+\log _{2} n\right)},
$$

which tends to 0 as long as $\alpha>2 / 5$.

By use of the remark following the proof of Lemma 3.2, the preceding corollary can be strengthened for unimodular orthogonal matrices to

Corollary 3.5. Let $\left(A_{n}\right)_{n \geq 1}$ be a sequence of unimodular orthogonal $n \times n$-matrices with $\left\|A_{n}: l_{2}^{n} \rightarrow l_{2}^{n}\right\| \leq 1$. Then $\mathbb{P}\left(\kappa\left(T \mid A_{n}\right) \geq n^{\alpha}\right) \rightarrow 0$ as $n \rightarrow \infty$ for any $\alpha>3 / 8$.

4. Hilbert space factorization. We start this section with the following easy observation.

Proposition 4.1. Let $T \in \mathcal{L}\left(l_{1}^{n}, l_{\infty}^{n}\right)$ be an operator induced by a matrix $\left(\tau_{i j}\right)$ with $\tau_{i j}= \pm 1$. Then

$$
\kappa_{n}\left(T: l_{1}^{n} \rightarrow l_{\infty}^{n}\right) \geq\left\|T: l_{2}^{n} \rightarrow l_{2}^{n}\right\|^{-1} n .
$$

Proof. Let $e_{1}, \ldots, e_{n}$ be the unit vectors in $l_{1}^{n}$. Then

$$
\sum_{k=1}^{n}\left\|e_{k}\right\|^{2}=n \quad \text { and } \quad \sum_{h=1}^{n}\left\|\sum_{k=1}^{n} \tau_{h k} T e_{k}\right\|^{2}=\sum_{h=1}^{n} \max _{1 \leq j \leq n}\left|\sum_{k=1}^{n} \tau_{h k} \tau_{j k}\right|^{2}=n^{3}
$$

imply that $\kappa(T \mid T) \geq n$. Now a glance at the definition of $\kappa_{n}(T)$ proves the claim.

Estimates on norms of random matrices are well known. We need a special case of Lemma 4 from [BGN]:

Lemma 4.2. There exist constants $c_{1}$ and $c_{2}$ such that, for all $t>0$,

$$
\mathbb{P}\left(\left\|T: l_{2}^{n} \rightarrow l_{2}^{n}\right\| \geq c_{1} \sqrt{(1+t) n}\right) \leq \exp \left(-\left(t-c_{2}\right) n\right) .
$$

COROLlary 4.3. There exists a constant $\chi>0$ such that

$$
\mathbb{P}\left(\kappa_{n}(T) \leq \chi \sqrt{n}\right) \leq e^{-3 n} .
$$


Proof. Set $t=3+4 c_{2}$ in the preceding lemma and define $\chi=$ $1 /\left(2 c_{1} \sqrt{1+c_{2}}\right)$. Then the lemma tells us that

$$
\mathbb{P}\left(\left\|T: l_{2}^{n} \rightarrow l_{2}^{n}\right\| \geq \sqrt{n} / \chi\right) \leq e^{-3\left(1+c_{2}\right) n} \leq e^{-3 n} .
$$

Now the assertion follows from the above proposition.

It can be immediately seen from Corollaries 3.3 and 4.3 that there cannot exist a sequence $\left(A_{n}\right)$ of $n \times n$-matrices with $\left\|A_{n}: l_{2}^{n} \rightarrow l_{2}^{n}\right\| \leq 1$ which is universal for the sequence of ideal norms $\kappa_{n}$. We prefer here to give a more quantitative statement which also implies Theorem 1.3.

THEOREM 4.4. Let $\left(A_{n}\right)_{n \geq 1}$ be a sequence of $n \times n$-matrices with $\| A_{n}$ : $l_{2}^{n} \rightarrow l_{2}^{n} \| \leq 1$. Then $\mathbb{P}\left(\kappa_{n}(\bar{T}) \geq n^{\beta} \kappa\left(T \mid A_{n}\right)\right) \rightarrow 1$ as $n \rightarrow \infty$ for any $\beta<1 / 10$. If the $A_{n}$ are unimodular orthogonal matrices then this even holds for $\beta<1 / 8$.

Proof. Since

$$
\mathbb{P}\left(\kappa_{n}(T) \geq n^{\beta} \kappa\left(T \mid A_{n}\right)\right) \geq 1-\mathbb{P}\left(\kappa_{n}(T) \leq \chi \sqrt{n}\right)-\mathbb{P}\left(\kappa\left(T \mid A_{n}\right) \geq \chi n^{1 / 2-\beta}\right),
$$

this follows from Corollaries 3.4, 3.5 and 4.3.

5. The size of universal sets of matrices. For $n \geq 1$, let $\mathcal{M}_{n}^{2}$ be the set of all $n \times n$-matrices with operator norm on $l_{2}^{n}$ less than or equal to 1 . We say that a sequence $\left(M_{n}\right)$ of subsets $M_{n} \subset \mathcal{M}_{n}^{2}$ is universal for $\kappa_{n}$ if there exists $c>0$ such that

$$
\kappa_{n}(T) \leq c \sup _{A_{n} \in M_{n}} \kappa\left(T \mid A_{n}\right) \quad \text { for } n=1,2, \ldots \text { and all operators } T .
$$

In this section, we want to clarify what size the members $M_{n}$ of a universal sequence for $\kappa_{n}$ must have. We first show an upper bound using entropy estimates. With the help of the results from the preceding sections we then derive a lower bound which is essentially the same.

THEOREM 5.1. There exists a universal sequence $\left(M_{n}\right)$ for $\kappa_{n}$ for which $\left|M_{n}\right| \leq 2^{n^{2}\left(5+\log _{2} n\right)}$ for $n=1,2, \ldots$

Proof. $M_{n}$ is going to be a $1 / 2$-net in $\mathcal{M}_{n}^{2}$. Let us first see that such a sequence is indeed universal for $\kappa_{n}$.

Given $B_{n}=B_{n}^{(0)} \in \mathcal{M}_{n}^{2}$, we inductively choose $A_{n}^{(k)} \in M_{n}$ such that $B_{n}^{(k+1)}=B_{n}^{(k)}-2^{-k} A_{n}^{(k)}$ satisfies $\left\|B_{n}^{(k+1)}: l_{2}^{n} \rightarrow l_{2}^{n}\right\| \leq 2^{-k-1}$. Then

$$
B_{n}=\sum_{k=0}^{\infty} 2^{-k} A_{n}^{(k)} \text {, }
$$


and it easily follows from the definition of $\kappa\left(T \mid A_{n}\right)$ that

$$
\kappa\left(T \mid B_{n}\right) \leq \sum_{k=0}^{\infty} 2^{-k} \kappa\left(T \mid A_{n}^{(k)}\right) \leq 2 \max _{A_{n} \in M_{n}} \kappa\left(T \mid A_{n}\right),
$$

showing the required inequality.

It remains to prove that there is a $1 / 2$-net $M_{n}$ in $\mathcal{M}_{n}^{2}$ with $\left|M_{n}\right| \leq$ $2^{2 n^{2}\left(5+\log _{2} n\right)}$. To this end, we identify $\mathcal{M}_{n}^{2}$ with a subset of $\mathbb{C}^{n^{2}}$ in the obvious way. Using the well known estimate

$$
\left\|A_{n}: l_{2}^{n} \rightarrow l_{2}^{n}\right\| \leq\left(\sum_{h=1}^{n} \sum_{k=1}^{n}\left|\alpha_{h k}\right|^{2}\right)^{1 / 2}
$$

for an $n \times n$-matrix $A_{n}=\left(\alpha_{h k}\right)$, we see that it is enough to find a $1 / 2$-net in euclidian norm with the required cardinality.

Pick now a point $x_{1}$ in $\mathcal{M}_{n}^{2}$. Assume that we have found points $x_{1}, \ldots, x_{k}$ in $\mathcal{M}_{n}^{2}$ such that $\left\|x_{i}-x_{j}\right\| \geq 1 / 2$ for $i \neq j$, where $\|\cdot\|$ now denotes euclidian norm. If possible, we choose a further point $x_{k+1} \in \mathcal{M}_{n}^{2}$ with $\left\|x_{k+1}-x_{i}\right\| \geq$ $1 / 2$ for $i=1, \ldots, k$. This process eventually stops leaving us with points $x_{1}, \ldots, x_{K} \in \mathcal{M}_{n}^{2}$ such that $\left\|x_{i}-x_{j}\right\| \geq 1 / 2$ for $i \neq j$ and such that the union of the balls with centers $x_{i}$ and radius $1 / 2$ covers $\mathcal{M}_{n}^{2}$. Hence the set $M_{n}=\left(x_{1}, \ldots, x_{K}\right)$ is indeed a $1 / 2$-net in euclidian norm.

Moreover, since also

$$
\left(\sum_{h=1}^{n} \sum_{k=1}^{n}\left|\alpha_{h k}\right|^{2}\right)^{1 / 2} \leq \sqrt{n}\left\|A_{n}: l_{2}^{n} \rightarrow l_{2}^{n}\right\|,
$$

we find that $\mathcal{M}_{n}^{2} \subset \sqrt{n} B$ where $B$ is the euclidian unit ball in $\mathbb{C}^{n^{2}}$. Hence the balls with centers $x_{i}$ and radius $1 / 4$ are contained in $(\sqrt{n}+1 / 4) B$. They are also mutually disjoint, so volume comparison yields

$$
K\left(\frac{1}{4}\right)^{2 n^{2}} \leq\left(\sqrt{n}+\frac{1}{4}\right)^{2 n^{2}}
$$

which finally implies

$$
\left|M_{n}\right|=K \leq(4 \sqrt{n}+1)^{2 n^{2}} \leq 2^{n^{2}\left(5+\log _{2} n\right)} .
$$

THEOREM 5.2. If the sequence $\left(M_{n}\right)$ is universal for $\kappa_{n}$ then there exists $\alpha>0$ such that $\left|M_{n}\right| \geq 2^{\alpha n^{2}}$ for $n=1,2, \ldots$

Proof. Assume that $\left(M_{n}\right)$ is universal for $\kappa_{n}$ with constant $c>0$. We may also assume that each $M_{n}$ is finite. In particular,

$$
\kappa_{n}(T) \leq c \max _{A_{n} \in M_{n}} \kappa\left(T \mid A_{n}\right) \quad \text { for all } T \in \mathcal{M}_{n} .
$$


It then follows from Corollary 4.3 and Theorem 3.1 that

$$
\begin{aligned}
1-e^{-3 n} & \leq \mathbb{P}\left(\kappa_{n}(T)>\chi \sqrt{n}\right) \leq \mathbb{P}\left(\max _{A_{n} \in M_{n}} \kappa\left(T \mid A_{n}\right)>(\chi / c) \sqrt{n}\right) \\
& \leq\left|M_{n}\right| 2^{-2^{-33}} \chi^{10} n^{2} / c^{10}+4 n\left(4+\log _{2} n\right)
\end{aligned}
$$

This easily gives the lower bound on $\left|M_{n}\right|$ as claimed in the theorem.

6. Application to some other ideal norms. In this section, we make use of the notation of $[\mathrm{PW}]$. The reader not familiar with this notation is advised to consult this reference for further information. We briefly review some things we need here.

$\mathcal{E}_{n}=\left(e_{1}, \ldots, e_{n}\right)$ stands for the first $n$ trigonometric characters $e_{k}(t)=$ $\exp (2 \pi i k t)$ in $L_{2}(0,1) . \mathcal{W}_{n}=\left(w_{1}, \ldots, w_{n}\right)$ stands for the first $n$ Walsh functions in $L_{2}(0,1)$ in some natural ordering. $W_{2^{n}}$ is the $n+1$-st HadamardWalsh matrix inductively defined by

$$
W_{1}=\frac{1}{\sqrt{2}}\left(\begin{array}{ll}
+1 & +1 \\
+1 & -1
\end{array}\right) \quad \text { and } \quad W_{2^{n+1}}=\frac{1}{\sqrt{2}}\left(\begin{array}{ll}
+W_{2^{n}} & +W_{2^{n}} \\
+W_{2^{n}} & -W_{2^{n}}
\end{array}\right) .
$$

The matrices $E_{n}$ were already defined in the introduction.

The $n$th trigonometric and Walsh type norms of an operator $T$ are denoted by $\varrho\left(T \mid \mathcal{E}_{n}, \mathcal{I}_{n}\right)$ and $\varrho\left(T \mid \mathcal{W}_{n}, \mathcal{I}_{n}\right)$, respectively. Given $T$, we let

$$
\begin{aligned}
\varrho\left(T \mid \mathcal{E}_{n}^{\max }, \mathcal{I}_{n}\right) & =\max \{\varrho(T \mid \mathcal{E}(F), \mathcal{I}(F)): F \subset N \text { and }|F| \leq n\}, \\
\varrho\left(T \mid \mathcal{W}_{n}^{\max }, \mathcal{I}_{n}\right) & =\max \{\varrho(T \mid \mathcal{W}(F), \mathcal{I}(F)): F \subset N \text { and }|F| \leq n\} .
\end{aligned}
$$

We now state the main theorem of this section.

THEOREM 6.1. None of the following sequences of ideal norms is uniformly equivalent to the sequence $\kappa_{n}$ :

$$
\kappa\left(E_{n}\right), \quad \varrho\left(\mathcal{E}_{n}, \mathcal{I}_{n}\right), \quad \varrho\left(\mathcal{E}_{n}^{\max }, \mathcal{I}_{n}\right), \quad \varrho\left(\mathcal{W}_{n}, \mathcal{I}_{n}\right), \quad \varrho\left(\mathcal{W}_{n}^{\max }, \mathcal{I}_{n}\right) .
$$

Moreover the sequence $\kappa\left(W_{2^{n}}\right)$ is not uniformly equivalent to the sequence $\kappa_{2^{n}}$.

Proof. It is shown in [Bou2] that $\varrho\left(\mathcal{W}_{n}^{\max }, \mathcal{I}_{n}\right) \leq c \varrho\left(\mathcal{W}_{n}, \mathcal{I}_{n}\right)$ with $c$ independent of $n$ (see also $[\mathrm{PW}]$, p. 326). This means that the sequences $\varrho\left(\mathcal{W}_{n}^{\max }, \mathcal{I}_{n}\right)$ and $\varrho\left(\mathcal{W}_{n}, \mathcal{I}_{n}\right)$ are uniformly equivalent. We conclude from Theorem 1.3 that the sequence $\kappa\left(W_{2^{n}}\right)$ is not uniformly equivalent to the sequence $\kappa_{2^{n}}$. Since $\varrho\left(\mathcal{W}_{2^{n}}, \mathcal{I}_{2^{n}}\right)=\kappa\left(W_{2^{n}}\right)$, we conclude that neither $\varrho\left(\mathcal{W}_{n}, \mathcal{I}_{n}\right)$ nor $\varrho\left(\mathcal{W}_{n}^{\max }, \mathcal{I}_{n}\right)$ can be uniformly equivalent to $\kappa_{n}$.

As already observed in [Hin], the methods of [Bou2] imply that there exists $c>0$ such that $\varrho\left(\mathcal{E}_{n}^{\max }, \mathcal{I}_{n}\right) \leq c \sqrt{1+\log n} \varrho\left(\mathcal{E}_{n}, \mathcal{I}_{n}\right)$. Since the sequence $\varrho\left(\mathcal{E}_{n}, \mathcal{I}_{n}\right)$ is uniformly equivalent to the sequence $\kappa\left(E_{n}\right)$ (see [PW], p. 285), we infer from Theorem 1.3 that none of the sequences $\kappa\left(E_{n}\right), \varrho\left(\mathcal{E}_{n}, \mathcal{I}_{n}\right)$, and $\varrho\left(\mathcal{E}_{n}^{\max }, \mathcal{I}_{n}\right)$ is uniformly equivalent to $\kappa_{n}$. 


\section{References}

[BGN] G. Bennett, V. Goodman and C. M. Newman, Norms of random matrices, Pacific J. Math. 59 (1975), 359-365.

[Bou1] J. Bourgain, A Hausdorff-Young inequality for B-convex Banach spaces, ibid. 101 (1982), 255-262.

[Bou2] -, Vector-valued Hausdorff-Young inequalities and applications, in: Lecture Notes in Math. 1317, Springer, 1988, 239-249.

[DF] A. Defant and K. Floret, Tensor Norms and Operator Ideals, North-Holland, Amsterdam, 1993.

[Hin] A. Hinrichs, Type norms with respect to characters of compact abelian groups and algebraic relations, Arch. Math. (Basel) 68 (1997), 265-273.

[Koe] H. König, On the Fourier-coefficients of vector-valued functions, Math. Nachr. 152 (1991), 215-227.

[Kwa] S. Kwapień, Isomorphic characterizations of inner product spaces by orthogonal series with vector valued coefficients, Studia Math. 44 (1972), 583-595.

[Pee] J. Peetre, Sur la transformation de Fourier des fonctions à valeurs vectorielles, Rend. Sem. Mat. Univ. Padova 42 (1969), 15-26.

[Pis] G. Pisier, Factorization of Linear Operators and Geometry of Banach Spaces, CBMS Regional Conf. Ser. in Math. 60, Amer. Math. Soc., Providence, 1986.

[PW] A. Pietsch and J. Wenzel, Orthonormal Systems and Banach Space Geometry, Cambridge Univ. Press, 1998.

[Tom] N. Tomczak-Jaegermann, Banach-Mazur Distances and Finite-Dimensional Operator Ideals, Longman, Harlow, 1989.

Mathematisches Institut

FSU Jena

D-07743 Jena, Germany

E-mail: nah@rz.uni-jena.de
Current address:

Department of Mathematics

Texas A\&M University College Station, TX 77843, U.S.A. 